\title{
The Shaping of a National Ignition Campaign Pulsed Waveform
}

G. Brunton, G. Erbert, D. Browning, E. Tse

July 28, 2011

IAEA 8th Technical Conference San Francisco, CA, United States June 20, 2011 through June 24, 2011 
This document was prepared as an account of work sponsored by an agency of the United States government. Neither the United States government nor Lawrence Livermore National Security, LLC, nor any of their employees makes any warranty, expressed or implied, or assumes any legal liability or responsibility for the accuracy, completeness, or usefulness of any information, apparatus, product, or process disclosed, or represents that its use would not infringe privately owned rights. Reference herein to any specific commercial product, process, or service by trade name, trademark, manufacturer, or otherwise does not necessarily constitute or imply its endorsement, recommendation, or favoring by the United States government or Lawrence Livermore National Security, LLC. The views and opinions of authors expressed herein do not necessarily state or reflect those of the United States government or Lawrence Livermore National Security, LLC, and shall not be used for advertising or product endorsement purposes. 


\title{
The Shaping of a National Ignition Campaign Pulsed Waveform
}

\author{
Gordon Brunton, Gaylen Erbert, Don Browning, Eddy Tse
}

\section{Lawrence Livermore National Laboratory, Livermore, CA. 94550}

The National Ignition Facility (NIF) at Lawrence Livermore National Laboratory is a stadium-sized facility containing a 192 beam, 1.8 megajoule, 500 terawatt ultraviolet laser system used for inertial confinement fusion research. For each experimental shot, NIF must deliver a precise amount of laser power on the target for successful and efficient target ignition, and these characteristics vary depending on the physics of the particular campaign. The precise temporal shape, energy and timing characteristics of a pulsed waveform target interaction are key components in meeting the experimental goals. Each NIF pulse is generated in the Master Oscillator Room (MOR) using an electro-optic modulator to vary the intensity of light in response to an electrical input. The electrical drive signal to the modulator is produced using a unique, high-performance arbitrary waveform generator (AWG). This AWG sums the output of 140 electrical impulse generators, each producing a 300ps pulse width Gaussian signal separated in time by $250 \mathrm{ps}$. By adjusting the amplitudes and summing the 140 impulses, a pulsed waveform can be sculpted from a seed 45ns square pulse. Using software algorithms written for NIF's Integrated Computer Control System (ICCS), the system is capable of autonomously shaping 48 unique experimental pulsed waveforms for each shot that have demonstrated up to $275: 1$ contrast ratio with $\pm 3 \%$ absolute error averaged over any 2 ns interval, meeting the stringent pulse requirements needed to achieve ignition. In this paper, we provide an overview of the pulse shaping system, software algorithms and associated challenges that have been overcome throughout the evolution of the controls.

Key word: pulse shaping, laser controls

\section{Introduction}

The NIF laser system [1] provides a scientific center for the study of inertial confinement fusion (ICF) and matter at extreme energy densities and pressures. One of the many tunable parameters critical to the success of ICF experiments is the laser pulse shape and timing [2]. Each NIF Quad (4 beams) can be configured, through experimental specifications, with distinct temporal pulse shapes. Once configured, controlling the pulse shape and energy within tight tolerances is critical to meeting these experimental goals [3]. NIF's Integrated Computer Control System (ICCS) [4] software algorithms have been developed to autonomously create and regulate each of these pulse shapes using a high bandwidth, programmable arbitrary waveform generator (AWG) to sculpt the required waveform from a seed optical square pulse.

Since the pulse shaping systems' inception the software algorithms have evolved considerably to meet the increasingly challenging pulse shape characteristics. In this paper we describe an overview of the pulse shaping system and the software controls used to successfully meet a wide variety of NIF experiment goals.

\section{Pulse Shaping System}

Each of the NIFs' 48 Quads is capable of generating a unique temporal pulse shape to support the experimental goals. Each pulse shape is sculpted from a $45 \mathrm{~ns}$ square optical pulse using an arbitrary waveform generator (AWG). The AWG utilizes a 2-stage lithium niobate electro-optic modulator that varies the intensity of light in response to an electrical input. The electrical drive signal to the modulator is produced by a unique, high-performance AWG. The AWG was created by Highland Technologies, Inc. specifically for the NIF and provides programmable 16 bit amplitude modulation control over an Ethernet protocol. Each AWG is deployed in a self-contained replaceable unit named an Amplitude Modulator Chassis (AMC). All of the 48 AMCs are physically located in Master Oscillator Room (MOR) of the Injection Laser
System (ILS) [5] section of the NIF. Figure 1 below shows a block diagram for the system components of an AMC.

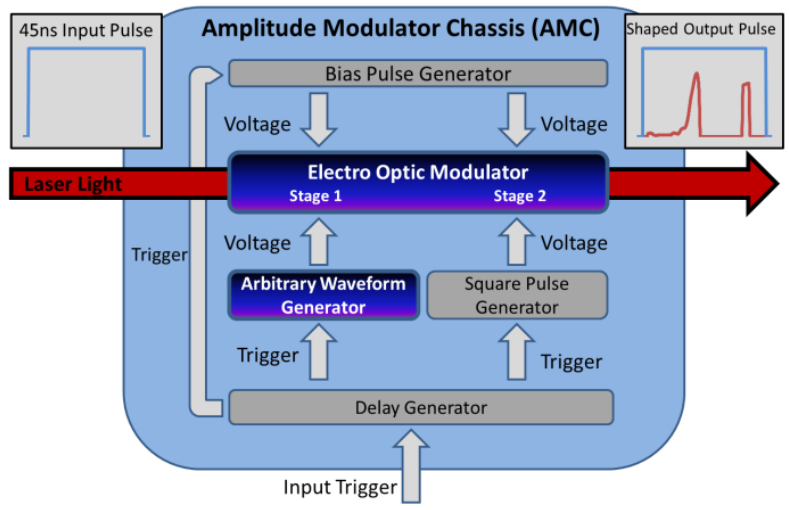

Figure 1 - Amplitude Modulator Chassis System Components

The AWG sums the output of 140 electrical impulse generators, each having 300ps pulse widths and temporal separation of 250ps. By adjusting the amplitudes and summing the 140 Gaussian pulses, an arbitrary electrical pulse shape is generated. An additional phase modulator is then driven with a square pulse generator to sharpen the pulse edges and regulate the output energy of the pulse shape. Figure 2 below depicts an example of how an arbitrary waveform is generated by the system using the summation of the Gaussian impulse generators. The following sections describe the software controls developed to autonomously shape each of the experimental goal waveforms using a close loop feedback control process. 


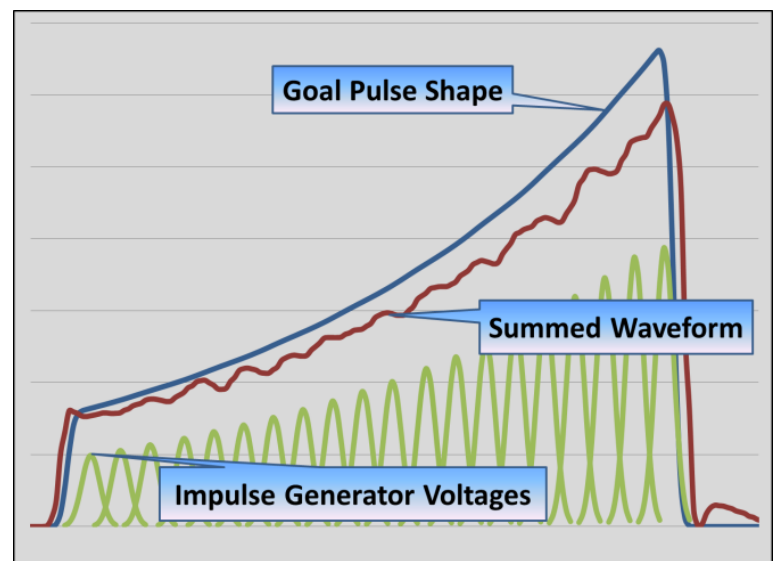

Figure 2 - Example Pulse Generation using Impulse Summation

\section{Pulse Shaping Controls}

NIF pulse shaping was originally accomplished using a LabView based system requiring operator interaction to manually shape the desired experimental waveform. This process relied heavily on human interaction and judgment and took many hours to complete. It was obvious that this process would not scale to a full NIF 48 Quad system and as such led to the design and implementation of the pulse shaping control system. The deployment of the controls has resulted in a completely autonomous system capable of shaping an arbitrary goal waveform concurrently on all NIF Quads within one hour of execution time. Figure 3 below shows a block diagram depicting the main software components involved in the autonomous shaping of a NIF pulse.

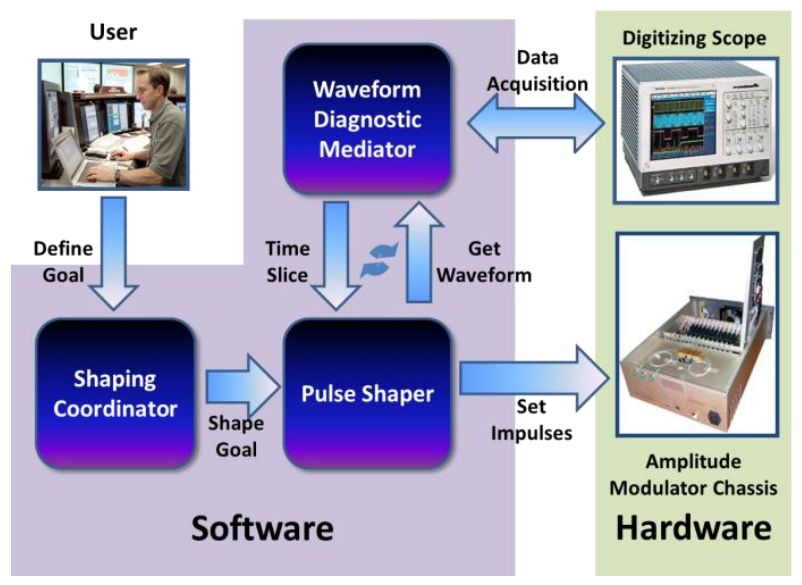

Figure 3 - Main Pulse Shaping Software Components

\subsection{Shaping Coordinator}

Each NIF shot calls for up to 48 Quads to participate in achieving the experiment goals. The Shaping Coordinator establishes and mediates a session of Pulse Shapers corresponding to the Quads required for the shot. The coordinator initiates autonomous operations that allow concurrent execution of actions, such as shaping or energy regulation, on an established session.

\subsection{Scope Diagnostic Mediator}

The pulse shaping close loop feedback controls use scope digitizers to capture the generated optical waveform. To minimize system costs and space requirements, all 48 Quads are shaped using a total of 3 scope digitizers (16 AMC waveforms per scope). The Scope Diagnostic Mediator restricts the use of a scope to one Pulse Shaper at any particular time. The mediator allocates each Pulse Shaper a time slice in a prioritized round robin manner.

\subsection{Pulse Shaper}

In order to achieve the experimental goals, each Quad (and thus each AMC) often require a different pulse shape specification. The Pulse Shaper control is responsible for performing the autonomous close loop feedback control to iteratively shape each Quad waveform to the goal pulse shape profile.

Each of these software components play a vital role in the waveform sculpting process however the rest of this paper focuses predominantly on the Pulse Shaper control as it provided the greatest challenges. The shaping process consists of several sequential phases starting with the calibration of the system, followed by shaping the pulse, and completing with the verification of the waveform and regulating of the pulse shape and energy over the duration of the experimental shot.

\section{Impulse Calibration}

Calibration of each AMC, prior to pulse shaping, is critical to achieving the precision requirements required for an ICF waveform. Each AMC system is required to support waveforms from $0.1 \mathrm{~ns}$ to $30 \mathrm{~ns}$ in pulse duration, with contrast ratios of up to 275:1 and timing precision Quad to Quad of 30ps RMS. A major factor affecting waveform variance over time is drift in the timing of each AWG impulse. The impulse calibration of each AWG originally involved an intensive two day procedure requiring a specialized system configuration and manual tuning of each individual impulse location.

Software controls have been developed to perform this calibration autonomously without the need for specialized system configurations. The calibration operation segments the 140 AMC impulses into four sets, each comprising of every fourth impulse. Selecting every fourth impulse in a calibration set maximizes the execution parallelism while ensuring there is no influencing overlap in signal summation. The execution of the calibration configures an equal voltage on each impulse, performs a high resolution (1ps per point) waveform acquisition, measures the time of each peak by interpolation of the full width, half maximum Gaussian at the impulse location, and updates the impulse offsets to move the peaks to their expected location. This iterative process continues until all peaks in the set are within a configurable tolerance of their expected peak locations (maximum of $\pm 1.8 \mathrm{ps}$ per impulse and mean of $\pm 0.9 \mathrm{ps}$ for the entire impulse set). An identical process is then performed on the other three sets of impulses.

The automation of the impulse calibrations has reduced the overall execution time from a two day activity to an average of 15 minutes with similar precision results. Due to this significantly reduced duration, calibrations are now scheduled at regular maintenance intervals ensuring continuous consistent system performance. Figure 4 diagram below depicts an example of the 'picket fence' waveform used in the calibration of each of the impulse set. 


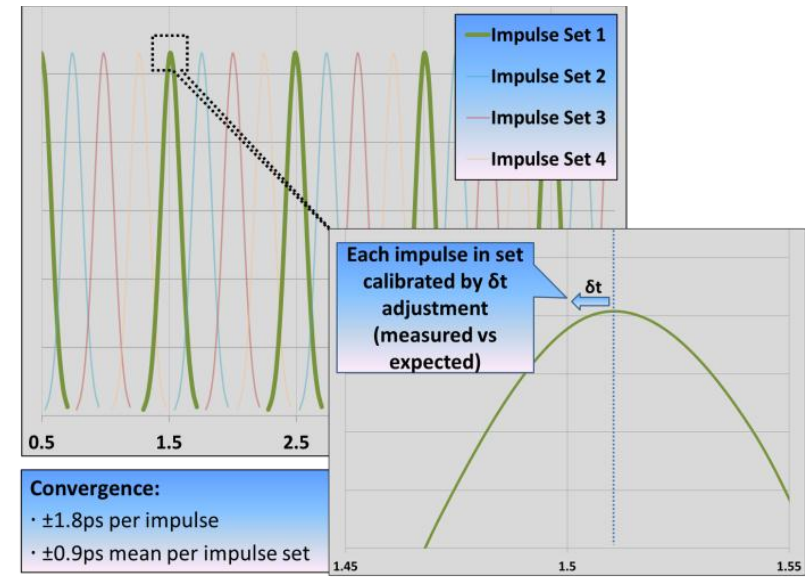

Figure 4 - Example Impulse Calibration Waveform Sets

\section{Carving the Shape}

The waveform shaping activity commences with the specification of the desired waveform. The specification consists of a 700 point, two dimensional array of time and amplitude pairs defining the goal shape. The shape can consist of either a single or double pulse. In addition, the specification defines the start and end times of the square pulse modulators used to sharpen the pulse edges to a rise or fall duration of 100 ps.

To ensure each AMC uses a consistent time reference the shaping process commences by enabling voltage on the first AWG impulse. Linear interpolation, full width/half maximum, of the measured impulse peak defines the 'time zero' reference offset that all of the remaining impulses are relative to. Calibrating the location of the first impulse ensures that the adjustments of relative impulses based on measured power response are accurately aligned.

The shaping of a pulse is iterative by nature. An iterative close loop adjustment process was chosen to provide a system capable of robustly coping with noise and calibration inaccuracies. Assuming a linear power response, each iteration adjusts the impulse voltage based on the power error at the impulse location $j$ as follows:

$$
\begin{gathered}
V_{j}^{\prime}=V_{j} \cdot \frac{G_{j}}{M_{j}} \\
\Delta V_{j}=V_{j}^{\prime}-V_{j}=V_{j} \cdot\left(\frac{G_{j}}{M_{j}}-1\right)
\end{gathered}
$$

where, $V_{j}=$ Impulse voltage,

$$
G_{j}=\text { Goal power }
$$$$
M_{j}=\text { Measured power }
$$

As the optical response from an impulse voltage is actually non-linear (the actual response is approximately $\operatorname{sine}^{2}$ ) in addition to each impulse receiving a voltage contribution from its neighboring impulse signals (due to the width of the Gaussian signal) a gain factor $g$ is applied to each voltage delta to temper the adjustment step. The resulting voltage delta adjustment is:

$$
\Delta V_{j}=V_{j} \cdot\left(\frac{G_{j}}{M_{j}}-1\right) \cdot g
$$

This scaling factor is dynamic based on $\frac{G_{j}}{M_{j}}$ ratio thresholds and commences with a ratio of 0.25 . Based on these configurable parameters the iterative shaping process typically takes 10-20 iterations to shape any arbitrary waveform.
The square pulse modulators, used to achieve the sharp pulse rise and fall, are set prior to the commencement of pulse shaping. The benefit of setting these initially is that the modulation influence they have on the pulse shape is factored out while shaping rather than adjusting the shape once they are enabled. The consequence of enabling the modulators prior to shaping is that impulse voltages at the rise and fall of the pulse cannot be adjusted by power response alone as the modulators suppress a significant amount of the impulse signal which results in the above formula overdriving the impulse voltage and causing undesirable pulse modulation.

It is important to set these edge impulse voltages as their small summed signal contributions are required to avoid sag in the waveform which again results in pulse modulation when the remainder impulses attempt to compensate for the sag. As such the edge impulse voltages are initially set based on ratio of the linear extrapolation from the neighbor impulse goal amplitude. Figure 5 below depicts graphically how edge impulse voltages are initially set.

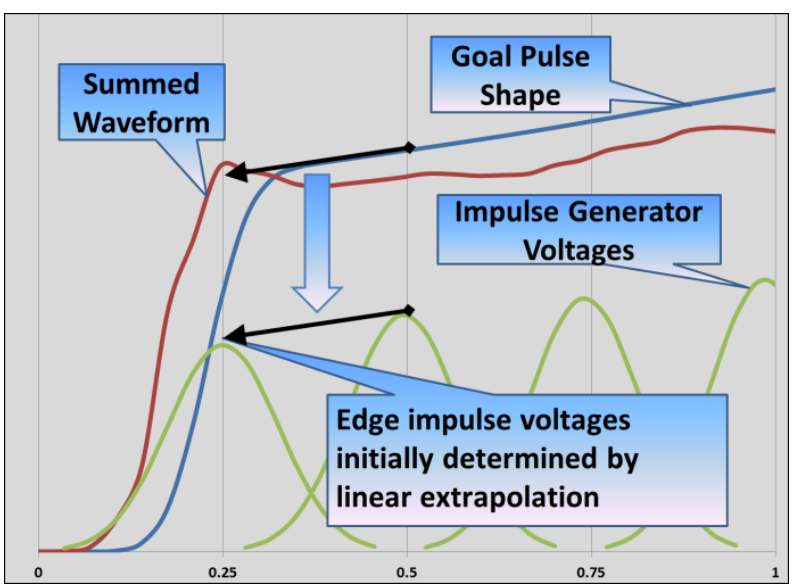

Figure 5 - Edge Impulse Voltage Extrapolation

Once the summed waveform amplitude error (measured vs. goal) is below a configurable tolerance (typically 10\%) the adjustment of the edge impulse voltages reverts to the same algorithm as the other impulses. These final few iterations assist in removing any residual pulse modulations at the peaks of the pulse. The impulse voltage adjustment algorithm described above details the default used for shaping pulses. The pulse shaping system uses an object oriented strategy pattern design [6] to allow alternate algorithms to be substituted in real-time should more appropriate adjustment methods be identified for specific goal pulse shapes. In addition to the default strategy, which adjusts impulse voltages based solely on the voltage response at the specific impulse time point, we have experimented with other strategies. Examples of alternate strategies experimented with include various windowed error algorithms (Hann, Hamming, Bartlett, etc). These strategies adjust the impulse voltage based on a weighted average error around the impulse location rather than the default single error measurement. Although these alternate shaping strategies provide marginally better results on specific pulse shapes the default adjustment strategy has proven to provide the best overall results.

\section{Timing the Pulse}

Precise timing of each pulse is critical to meeting the requirement of 30ps RMS for all pulses arriving at target chamber center. Accurately aligning the pulse edge and consistently repeating it shot to shot is very important in meeting this stringent requirement. As previously described, the sharp pulse edges are 
achieved by applying a square pulse modulator voltage to the AWG waveform. The square pulse modulator rapidly cancels the AWG signal in regions outside the square pulse signal thereby achieving a rise or fall time of 100ps which cannot be achieved with an impulse Gaussian alone.

To achieve reliable timing repeatability, the pulse shaping algorithms dynamically adjust the square pulse edge while shaping to minimize the error at the pulse rise and fall. Adjustments are performed to minimize error at the mid point of the rise or fall.

The error $E$ at the rise or fall is defined as the error sum between the measured waveform $M$ and the goal pulse $G$ at the two time points $\left(t_{j}, t_{j+1}\right)$ weighted to the time that brackets the half maximum amplitude $t_{x}$.

$$
E=\sum_{i=j}^{j+1} W_{i} \cdot D_{i}
$$

where weight $W_{i}$ and delta $D_{i}$ are defined,

$$
W_{i}=\frac{\left|t_{x}-t_{i}\right|}{t_{j+1}-t_{j}}, \quad D_{i}=M_{i}-G_{i}
$$
follows,

Using the weighted error the square pulse edge is adjusted as

$$
\Delta t=\frac{E}{m} \cdot c
$$

where, $t=$ Temporal edge adjustment,

$E=$ Edge error,

$m=$ Max gradient of edge

$c=$ Constant to convert to time units

Convergence of the edge adjustments occurs when the error $E$ is a) less than a configurable threshold (typically $1 \mathrm{~mW}$ ) and b) the waveform peak power is within a configurable threshold of its goal peak power (typically 10\%) as depicted below in Figure 6.

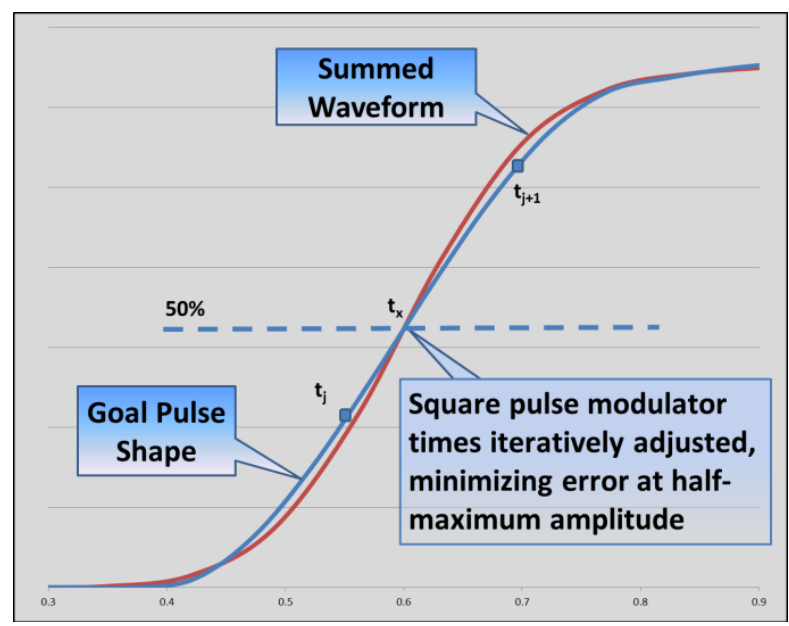

Figure 6 - Square Pulse Modulator Edge Correction

\section{Shaping Convergence}

The iterative shaping process continues until a defined convergence criterion has been satisfied. Similar to the shaping process, the convergence criterion is also a strategy based by design and can be dynamically changed at runtime. The default convergence strategy uses a standard deviation methodology. On each pulse shaping iteration the integral error $E$ between the measured waveform $M$ and the goal pulse $G$ over the entire pulse is calculated.

$$
E=\sum_{i=0}^{n} M_{i}-G_{i}
$$

Convergence is met when the standard deviation $\sigma$ of the previous six iteration integral errors is less than a configurable threshold (typically $1.0 \mathrm{~mW}$ ).

$$
\sigma=\sqrt{\frac{1}{n} \sum_{i=1}^{n}\left(E_{i}-\bar{E}\right)^{2}} \quad \text { where } n=6
$$

Measuring a minimal error standard deviation over a number of iteration nominally indicates that the shaping algorithm has achieved the best possible waveform. Several other convergence strategies have been implemented as part of the system design. These strategies typically enforce tighter convergence criteria and are normally used on isolated laser shots when quality requirements on the pulse shaper are higher.

Using a strategy based design for the pulse shaping algorithms has ensured a flexible model that adapts easily to the operational requirements placed on the system for any particular experimental goal.

\section{Pulse Verification}

After convergence of the pulse shaping algorithms the final stage of process verifies that the shaped waveform meets the experiment requirements. Convergence does not guarantee that the experiment goal has been met, it only measures that the pulse shape is the best it can achieve. As the shaping process occurs early in the experimental shot cycle the pulse verification is performed several times up until seconds prior to the firing of the laser shot. This ensures that the system confirms that the pulse has not drifted from the original pulse requirements. The verification effectively consists of a tolerance band around the pulse goal ensuring that each point of the pulse is within specification from both an amplitudinal and temporal perspective. All tolerances are defined as configuration items in the controls database.

\section{Continuous Pulse Regulation}

Several hours can elapse from the convergence of the shaping process to the execution of the final high powered system shot. A recent enhancement to the shaping algorithms has been commissioned to deal with small drifts in the pulse shape and energy over time. These pulse regulation additions maintain both the integrated pulse energy and pulse shape.

Pulse energy regulation continuously adjusts the AMC square pulse modulator voltage to maintain a constant integrated energy of the goal pulse $G$ versus the measured waveform $M$.

$$
\Delta V=\sum_{i=0}^{n}\left(G_{i}-M_{i}\right) \cdot c
$$

where, $\Delta V=$ Adjustment voltage delta,

$$
\begin{aligned}
& G_{i}=\text { Goal power } \\
& M_{i}=\text { Measured power } \\
& c=\text { Constant to convert to voltage units }
\end{aligned}
$$

In addition to energy regulation, shape regulation was recently added to address tilt in the seed square pulse over time. Shape regulation is performed in a similar manner to the actual shaping algorithms previously described with two major distinctions.

Firstly the AWG impulse voltages are only adjusted to maintain the shape if the present shape is verified to already be within tolerance bounds. This ensures that no gross modifications 
will be made to the shape for system safety reasons. These small tweaks to the shape over time ensure that drift, as a result of light tilt, is effectively eliminated. The second restriction to shape regulation is that no change to the square pulse modulator timing is performed. The system has historically experienced very little temporal drift and thus there has presently been no need to perform such corrections.

Implementing the pulse regulation enhancements has proven that the tight pulse requirements can be maintained in the summed waveform over many hours resulting in consistent experiment data from shot to shot.

\section{Conclusion}

In this paper, the control system and underlying software algorithms required to calibrate, shape, verify and regulate any arbitrary NIF experimental pulse shape are discussed. Real-time operation is facilitated by this extensible, databasedriven, close loop feedback system, which meets all the system requirements presently defined for current and future NIF experiments.

\section{Acknowledgement}

This work performed under the auspices of the U.S. Department of Energy by Lawrence Livermore National Laboratory under Contract DE-AC52-07NA27344.

\section{References}

1. E. I. Moses, "Overview of the National Ignition Facility", Fusion Science and Technology 54, pp. 361-366 (2008).

2. J. D. Lindl, B. A. Hammel, B. G. Logan, D. D. Meyerhofer, S. A. Payne, J. D. Sethian, "The US ICF Ignition Program and the Inertial Fusion Energy Program", 30th EPS Conference on Controlled Fusion and Plasma Physics, St. Petersburg, Russia, 2003

3. David H. Munro, Peter M. Celliers, Gilbert W. Collins, David M. Gold, Luiz B., Da Silva, Steven W. Haan, Robert C. Cauble, Bruce A. Hammel, Warren W. Hsing, "Shock timing technique for the National Ignition Facility", 42nd Annual Meeting of the American Physical Society Division of Plasma Physics with the 10th International Congress on Plasma Physics, Quebec City (CA), 2000

4. P.J. Van Arsdall, R.C. Bettenhausen, F.W. Holloway, R.A. Saroyan, J.P. Woodruff, "National Ignition Facility Integrated Computer Control System", Third Annual International Conference on Solid State Lasers for Application (SSLA) to Inertial Confinement Fusion (ICF), Monterey, CA, 1998

5. Mark Bowers, Scott Burkhart, Simon Cohen, Gaylen Erbert, John Heebner, Mark Hermann, Don Jedlovec, "The Injection Laser System on the National Ignition Facility", Solid State Lasers XVI: Technology and Devices, Proceedings of SPIE Vol. 6451, 2007

6. E. Gamma, R. Helm, R. Johnson, J. Vlissides: "Design Patterns, Elements of Reusable Object-Oriented Software", Addison Wesley, 1995. 